\title{
De l'onanisme à la masturbation. Une note historique
}

\author{
Georges ANDROUTSOS \\ Histoire de la Médecine, Faculté de Médecine, Université d'loannina, Grèce
}

\section{RESUME}

Cet article trace brièvement l'histoire de la masturbation. Plus particulièrement, sont abordés des sujets tels que la masturbation chez les anciens, l'attitude de l'Eglise à l'égard de cette pratique sexuelle, les accessoires facilitant la masturbation. Aussi sont présentées les diverses mesures de prévention de la masturbation utilisées autrefois, ainsi que les cures des conséquences de ce vice. En outre, sont également présentés les deux fameux ouvrages L'Onania et L'Onanisme, qui furent la justification de la polémique contre la masturbation.

Mots clés : masturbation, crime d'Onan, Tissot, Bekkers, objets masturbatoires, onanistes célèbres

\section{INTRODUCTION}

La masturbation est une auto-excitation des zones érogènes génitales en vue d'aboutir à l'orgasme. Cette pratique est une phase transitoire du développement normal de la fonction érotique dans l'espèce humaine [26]. Elle peut être solitaire, réciproque (homo ou hétérosexuelle), pratiquée en groupe (notamment pendant l'adolescence). La masturbation est également désignée par les mots autoérotisme (tendance à trouver la satisfaction sexuelle dans l'organe même où est apparu le besoin, sans l'intermédiaire d'un autre individu ou d'une image unifiée du moi. Dans l'auto-érotisme, seule est visée la satisfaction d'organe. Le corps et la vie sexuelle y fonctionnent de façon essentiellement fragmentaire), manipulation génitale (exploration tactile des organes génitaux chez l'individu lui-même ou chez autrui), onanisme (pratique masturbatoire. L'origine du mot est biblique. Onan s'adonnait au coït interrompu avec sa belle-sceur, enfreignant la loi du lévirat qui obligeait l'homme à donner une progéniture à la femme de son frère décédé). En réalité, alors que l'onaniste obtient son jouir par le coït interrompu (acte sexuel au cours duquel le partenaire masculin se dégage du vagin avant d'éjaculer, ceci dans un but anticonceptionnel ou pour des raisons psychopathologiques), le masturbé, lui, s'en tient exclusivement à l'empoigne mobile de sa main sur son sexe [10].

\section{GÉNÉRALITÉS}

En 1576, Montaigne dans l'Apologie de Raimond Sebond [Essais, Ch. 12, L. 2] vient à la relativité des valeurs morales et trace alors pour la première fois dans la langue française le mot "masturbation" [7].

Pendant plus d'un siècle le mot figurera sous deux formes qui coexisteront, manustupration (de manus = la main, et stupratio = action de souiller) et masturbation (du latin, masturbatio, ou du grec mastropeuein, mastropeuein = prostituer), avec tout de même un usage rare, voir confidentiel, car la masturbation n'est alors l'objet d'aucune condamnation.

On en trouve ensuite la trace en termes populaires ou métaphoriques dans la littérature du $17 \mathrm{e}$ siècle. Mais le mot n'est toujours pas dans les pages du dictionnaire : it brille par son absence dans la première édition du Dictionnaire de l'Académie, en 1694.

\section{LA MASTURBATION DANS L'ANTIQUITÉ}

Dans I' Égypte ancienne, pour participer à la vie de l'audelà, le défunt devait déclarer devant le tribunal divin qu'il ne s'était jamais livré à la masturbation [2]. Hermès avait initié Pan à la masturbation pour le consoler de la perte de la nymphe Echo. Hippocrate avait décrit les maux produits par l'abus des plaisirs, sous le nom de consomption dorsale. Aristote ne blâme pas les jeunes gens qui se masturbent, se contentant de les mettre en garde contre les "douleurs" provoquées par cette pratique. Diogène recommandait la masturbation. L'art précolombien nous a laissé un grand nombre d'œuvres représentant des sujets en train de se masturber (Figure 1). Dans l'ancienne Chine la masturbation était entièrement interdite à l'homme, car

\section{Correspondance :}

Dr. Georges ANDROUTSOS - 1 rue Ipeirou, 10433, Athènes, Grèce - Fax +302108235710 - Email lyon48@ otenet. $g r$ 


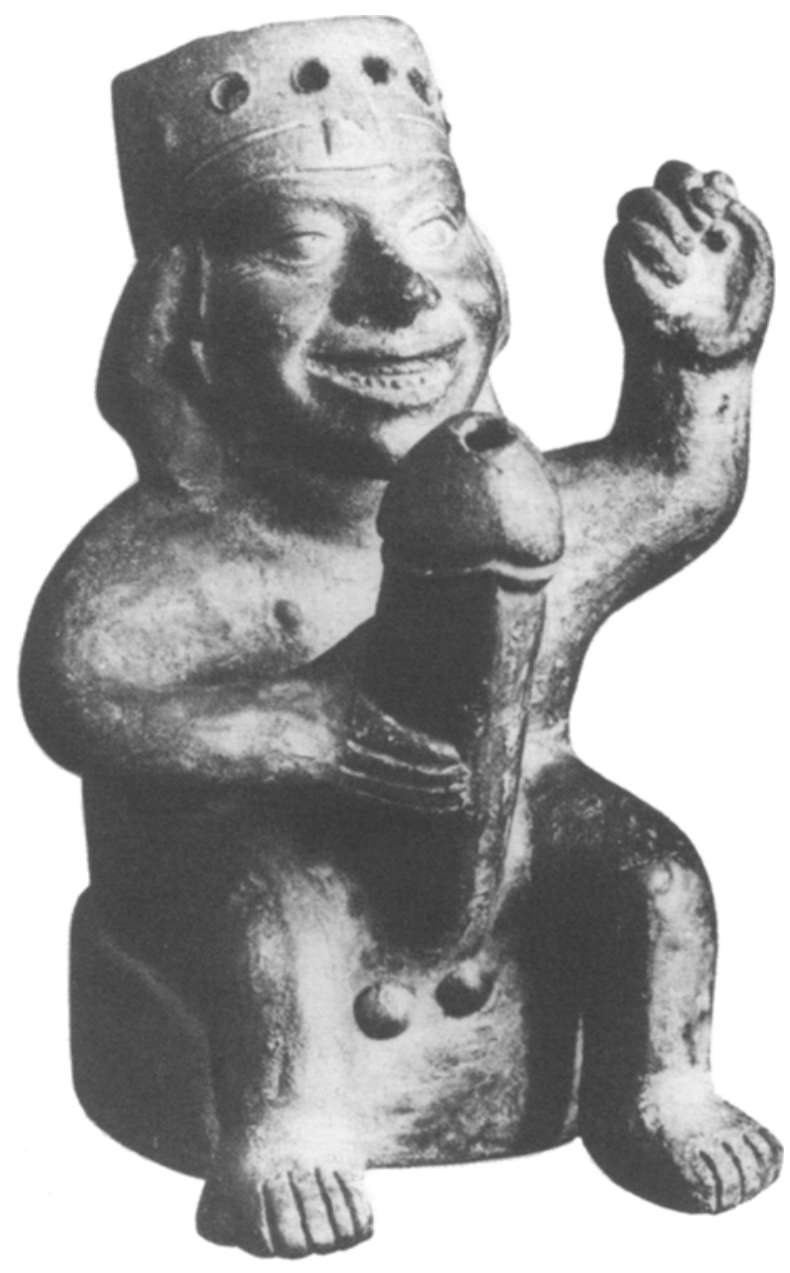

Figure 1 : Sujet en train de se masturber (Pérou, civilisation mochica).

elle entraînait une perte complète d'essence vitale [24]. On ne l'excusait que dans les cas où les circonstances particulières privaient un homme de compagnie féminine, et où la "semence dévitalisée" pourrait encrasser son organisme [17].

\section{L' ÉGLISE FACE A LA MASTURBATION}

Précocement la masturbation est considérée par l'Église comme un péché mortel et stipendiée comme un acte abominable. On sait combien le libertinage solitaire a suscité de tracas à la théologie chrétienne, tous les "cas" de conscience qu'il a posés aux clercs frustrés de partenaires. La répression fut ainsi longtemps affaire purement cléricale, ecclésiastique, aucune bruyante campagne "moralisatrice" anti-masturbatoire ne s'étant abattue sur les ouailles "séculières" pendant le Moyen Age ni la période classique. Donc la masturbation, dès le Moyen Age, est punie sévèrement mais selon des tarifs variables dans les pénitentiels. Saint Thomas analyse avec beaucoup d'indulgence la pollution nocturne, estimant qu'elle n'est jamais un péché, même si elle résulte de pensées lascives avant le sommeil, car, ditil, dans ce cas ce sont les pensées qui constituent un péché et non la pollution [18]. D'autres cependant sont beaucoup plus sévères, culpabilisant les victimes mais estimant toutefois que si elle résulte d'une trop longue abstinence, elle devient méritoire [20].

Etant donné l'importance qu'elle attachait à réprimer la masturbation, l'Église a cherché dans la Bible une justification à sa thèse et n'a pas hésité à déformer les faits dans ce but. La Genèse rapporte l'épisode d'Onan répandant sa semence sur le sol. On a cru - et on continue souvent à croire aujourd'hui que ce passage faisait allusion à la masturbation, et le terme d'onanisme est devenu synonyme de ce mot [34].

Les protestants étaient aussi opposés à la masturbation que les orthodoxes et les catholiques [25]. Luther déclarait : "Onan devait être un scélérat, malicieux et sans repentir. Son péché est des plus horribles. II est bien plus atroce que l'inceste et l'adultère. Nous l'appelons impudicité, oui, un péché de Sodome" [30].

\section{LE CRIME D'ONAN}

Dans l'Ancien Testament [Genèse, ch.37 et 38], sont relatés ainsi les faits : "La femme de Juda devint enceinte et enfanta un fils qu'elle appela Er. Elle devint encore enceinte et enfanta un fils qu'elle appela Onan. Elle enfanta encore un fils qu'elle appela Schéla. Juda prit pour Er, son premier-né, une femme nommée Tamar. Er était méchant aux yeux de l'Éternel ; et l'Éternel le fit mourir. Alors Juda dit à Onan : vas vers la femme de ton frère, prends-la comme beau-frère et suscite une postérité à ton frère. Onan, sachant que cette postérité ne serait pas à lui, se souillait sur terre lorsqu'il allait vers la femme de son frère, afin de ne pas donner de postérité à son frère. Ce qu'il faisait déplut à l'Éternel qui le fit aussi mourir. Alors Juda dit à Tamar, sa belle-fille : demeure veuve dans la maison de ton père jusqu'à ce que Schéla, mon fils, soit grand. II parlait ainsi dans la crainte que Schéla ne mourût comme ses frères ...".

Le mariage avait pour but la reproduction. Si elle n'était pas atteinte, l'union pouvait être dissoute. Dans un cas particulier, le mariage était pour l'homme une obligation : quand le frère aîné mourrait sans avoir laissé de descendant mâle, le frère cadet devait épouser la veuve. Cette forme de mariage obligatoire appelé lévirat (du latin levir = frère du mari), était prise fort au sérieux chez les Juifs. Mais elle était parfois ressentie très désagréablement par celui qui tombait sous le coup de cette loi, d'autant plus que les enfants nés de ce mariage étaient considérés comme les descendants légitimes du frère décédé. Onan refusait d'engrosser sa belle-sœur devenue veuve. II refusait d'obéir à la loi : de donner à son frère disparu une descendance, cela à son propre détriment puisque cet enfant né de lui se serait vu adjuger la part d'héritage devant revenif à Onan. Pour ne pas être dépossédé, il inventa tout simplement le coît interrompu : c'est-à-dire l'éjaculation hors du sexe féminin [22]. 
On conçoit la gravité du forfait d'Onan qui a commis un acte contre nature, sévèrement répréhensible et sévèrement réprimé. II ne ressort pas clairement du récit biblique s'il s'agit de masturbation ou de coitus interruptus. Ce qui est certain, c'est qu'il s'est retiré, qu'il a failli à sa mission génitrice.

Les opinions des théologiens et des exégètes sur ce qu'Onan faisait en réalité furent parfois divergentes. Certains estimaient qu'il se masturbait, d'autres qu'il pratiquait le coït interrompu. Quoi qu'il en soit, le refus d'Onan de donner une descendance à son frère lui valut le châtiment suprême, et son nom servit plus tard pour désigner la masturbation [23].

\section{LES PROTAGONISTES DE LA CAMPAGNE ANTI-MASTURBATOIRE}

Le médecin et hygiéniste suisse Simon Auguste David André Tissot (1728-1797) (Figure 2) est considéré comme le grand initiateur de la lutte contre la masturbation. Néanmoins il n'est que la répercussion de l'étonnement d'une Europe préscientifique qui découvre les mystères de la vie, avec la découverte du spermatozoïde par Anton van Leeuwenhoek en 1677, et y réagit de façon défensive par l'interdit de la masturbation. Et il s'agit ici d'un crime sexuel, transgressant le tabou suprême, celui de préservation de la vie.

Avant d'être un crime, un siècle plus tôt, la masturbation était considérée comme un simple péché. Aussi bien l'Église que la société supportaient la masturbation et dénonçaient simplement les excès du libertinage. La preuve de

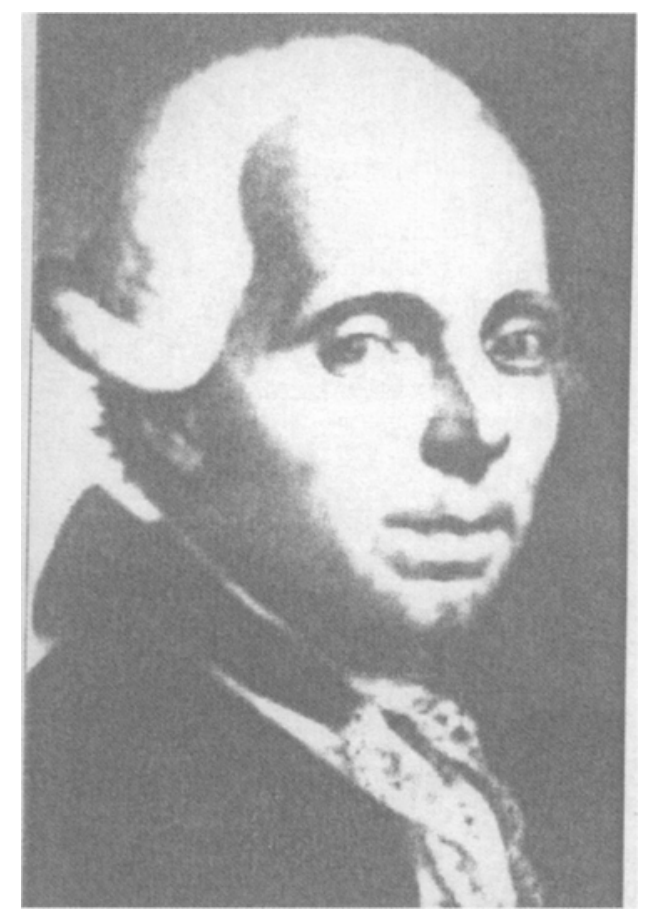

Figure 2 : Samuel Auguste Tissot (1728-1797). cette attitude à l'égard de la masturbation est le célèbre $D e$ la génération de l'homme ou tableau de l'amour conjugal [36] de Charles Patin (1633-1698) (médecin français, fils du doyen Gui Patin. II écrivit sous le pseudonyme de Nicolas Venette de la Rochelle), paru en 1685, qui ne fait aucune mention de ce mot.

En 1710, Bekker (s), un médecin anglais, publiait à Londres la brochure Onania ou le péché infâme de la souillure de soi et toutes ses conséquences affreuses chez les deux sexes, avec des conseils moraux et physiques à l'adresse de ceux qui ont déjà eu préjudice de cette abominable habitude [5].

Dans l'Onania [4] il n'y a que des observations ; toutes les réflexions de l'auteur ne sont que des trivialités théologiques et morales. Le style est à ce point agressif que la brochure connaît un succès immédiat et qu'elle fait l'objet de nombreuses rééditions. L'Onania était en fait une vaste supercherie éditée par un charlatan dans un but lucratif, car les conclusions de l'ouvrage invitaient le lecteur à se procurer à telle enseigne, une "teinture revigorante" et une "poudre prolifique", remèdes souverains contre ce penchant que l'on se procurait avec l'Onania chez le libraire habituel de Bekkers, pour la somme très modique de douze shillings.

Durant un demi-siècle, l'Onania connaît un rare succès de librairie. L'engouement du public ne s'explique que par l'effet de la nouveauté. Si de nombreux livres dénonçaient la débauche et l'adultère, aucun jusqu'alors ne s'était attaqué à l'impureté avec soi-même [20]. Peu de livres avaient fait autant de bruit depuis longtemps et son influence fut considérable. II en surgit le terme d'onanisme qui n'existait pas dans l'Encyclopédie. II atteignit non seulement le grand public mais aussi la profession médicale.

Tissot [35] a publié en 1758 à Lausanne son ouvrage Testament de morbis ex manustupratione (Essai sur les maladies produites par la masturbation) qui fut la justification de la croisade laïque contre la masturbation.

Deux ans plus tard, en 1760, la traduction française parut isolément : L'Onanisme, ou Dissertation physique sur les maladies produites par la masturbation. Cette publication réveilla les vieux démons de l'Inquisition et de la chasse aux sorcières et modifia durablement les attitudes et la morale sexuelle jusqu'au début du 20 e siècle. Ce livre symbole déclencha la plus grande répression sexuelle que l'Europe ait connu et terrorisa des générations de masturbateurs [37].

Le livre de Tissot contient beaucoup de citations et apporte la vaste expérience personnelle de l'auteur. Traduit en plusieurs langues, il sera maintes fois réédité et deviendra un grand classique, un traité de référence, influençant énormément la société et la médecine [28].

En 1895 Sigmund Freud publia son tableau morbide de la Névrose d'angoisse [13], qui reprenait à la lettre les symptômes que Bekkers et Tissot, attribuaient à la masturbation. Nous proposons la classification suivante des maux dont étaient atteints les malades anglais : 
1) affaiblissement des facultés intellectuelles, perte de mémoire, obscurcissement des idées, légère démence, angoisse continuelle, vertiges ;

2) les forces du corps manquent entièrement, arrêt de la croissance, somnolence, hypochondrie ou hystérie quasi constante ; tristesse, soupirs, palpitations, suffocations, défaillances, expectoration de matières calcaires, toux, fièvre lente, consomption ;

3) douleurs variables touchant la tête, la poitrine, l'estomac, les intestins, les articulations ; engourdissements douloureux de toutes les parties du corps ;

4) boutons sur le visage, pustules suppurantes sur la figure, dans le nez, sur la poitrine et les cuisses, démangeaisons continuelles, excroissances charnues sur le front ;

5) perte de l'érection, émission de sperme à la plus légère excitation ou lors de la défécation, écoulement continuel par l'urètre d'une sanie fétide ou d'une mucosité sale, priapisme douloureux, dysurie, brûlures mictionnelles, affaiblissement du jet, tumeurs des testicules, de la verge, de la vessie, impuissance et stérilité ;

6) troubles intestinaux : constipation, hémorroïdes, diarrhées, émission de glaires par l'anus [19].

Bekkers et Tissot avaient écrit leurs ouvrages dans le but commun de la répression physique et morale de pulsions sexuelles. Tissot recevait de partout des encouragements et des félicitations. Voltaire le remerciait ainsi : "Cet ouvrage est un service rendu au genre humain. Ma lettre serait plus longue si je pouvais m'abandonner à tous les sentiments d'estime que vous m'inspirez. Votre malade, Voltaire".

Rousseau en oublie pour la circonstance ses pratiques solitaires et exprime ainsi de bien coupables regrets : "Bien fâché de n'avoir pas connu plus tôt le Traité de la Manustupration ...Vous me dites que cet ouvrage a été prohibé à Paris, cela me consolerait de ce que le mien (Émile) y a été brûlé, si la sottise et la cafardise, en justifiant ce qu'elles blâment, ne montraient la honte et les misères de notre espèce...Je sais que nous sommes faits, vous et moi, pour nous entendre et pour nous aimer. Tous ceux qui pensent comme nous sont amis et frères ...Je suis à vos pieds, Monsieur" (juillet 1762).

Rousseau ne tarira jamais d'éloges pour "ce médecin de l'âme", comme il l'appelait et dont il aurait voulu faire son dernier confident : "Combien, dans ma dernière maladie, ne voudrais-je pas avoir un Tissot à mon chevet, afin que quand il n'y aurait plus rien à faire au corps, il fût encore le médecin de l'âme" (1er avril 1765) [7].

\section{ACCESSOIRES ÉROTIQUES FACILITANT LA MASTURBATION}

Dans les musées des prises de la Police Judiciaire, on trouve un grand nombre d'objets et accessoires utilisés par les masturbateurs. Aujourd'hui, ces accessoires et ces objets qui hantèrent les libertins et affranchis d'hier nous semblent anachroniques et désuets. Citons en exemple quelques uns :

Les masturbeuses (des mains qu'on prend dans la main qui les mancuvre, ou dans lesquelles on jouit par frottement) ; la main en or massif enrichie d'urne incrustée de pierreries pour recueillir le sperme ; la main cuir et peau (paume en cuir noir pour le tronc du pénis, pouce et index en pécari rose pour la calotte) ; la main d'écailles (une main féminine ravissante remplie de sable fin et gantée d'une robe fragile, diaprée, miroitante, empruntée à une espèce de poissons rares) ; l'étau à gland ou tenaille du sphinx : c'est un étau ordinaire d'atelier ou d'usine, mais revêtu d'un velours de pourpoint d'un rouge espagnol, lumineux, admirable ; son rôle : presser de droite et de gauche le gland, au moment de l'orgasme, afin de créer chez le patient les spasmes éjaculatoires souhaités ; la gueule de Sarah : une tête de lionne empaillée, mâchoire en bataille, gorge béante, qui servait aux éjaculations collectives ; la vespasienne de la Comtesse de ... (la copie grandeur nature de l'urinoir de la rue de la Croix des Petits Champs, avec ses inscriptions, ses demandes de rendezvous, ses petites annonces, ses gros mots, ses insultes, ses croûtons de pain laissés tout exprès par les dîneurs ; et, sculptée dans chaque paroi d'acier, au niveau des braguettes, une grotte contenant un récipient en forme de berceau pour récolter la semence virile des compagnons diurnes et nocturnes) [31].

\section{PRÉVENTION DE LA MASTURBATION}

La psychose des pertes séminales fut telle qu'elle donna lieu à des mesures innombrables contre ce "vice". II faudrait un tome pour dresser l'inventaire des procédés punitifs et des moyens de prévention mis en œuvre par les adultes pour lutter contre la masturbation des enfants mâles et (à un degré moindre) des filles [12].

Au cours du $19 \mathrm{e}$ siècle, la prévention de la masturbation fut prônée avec un tel enthousiasme qu'il faut bien soupçonner, chez les auteurs d'écrits contre l'onanisme et les inventeurs d'appareils destinés à le prévenir, un complexe d'onanisme non résolu ou une forme de sadisme fixée sur cette pratique [27].

On a l'impression, assurément excessive, d'une vaste coalition des parents, éducateurs, enseignants, confesseurs, sous la houlette des médecins, pour espionner les possibles victimes du vice solitaire, le prévenir et pallier ses désastreux effets. Tout semble ainsi suspect de pouvoir provoquer la pernicieuse habitude. En l'absence même de l'initiation criminelle par des camarades ou des aîné(e)(s), il faut se méfier de ce qui pourrait inciter à la découverte spontanée...[9].

Qu'elle s'opère au boudoir ou qu'elle se pratique à la dérobée, hors de portée du regard des parents, la masturbation est atrocement mal reçue. C'est qu'on la juge abominable. Associée non seulement à l'idée de scandale mais aussi (et surtout) à celle de malédiction, elle fait quasiment l'objet 
d'une chasse aux sorcières. Elle est le deuxième péché originel [32].

Parmi les remèdes aux vices solitaires, les vertus de la parole ont toujours constitué un antidote à la culpabilité. C'est l'aveu, rendu nécessaire par le flagrant délit. Le coupable avoue par désir de survivre! Comme tout était bon pour condamner, tout sera bon pour guérir. L'une des premières armes thérapeutiques sera le spectre de la déchéance que l'on agitait devant les yeux des délinquants du sexe. Viendront ensuite des potions, des onguents, des médications, des règles hygiéniques, et puis des appareils, des armures préventives, des systèmes protecteurs, des préservatifs, des garde-fous. Enfin, les grands moyens : la chirurgie, l'exérèse, l'ablation [7].

Parmi les remèdes on peut citer les calmants, les tempérants, les refroidissants, les antispasmodiques. On préconise les narcotiques, les hypnotiques et les somnifères qui endorment l'amour. C'est la mandragore, la belladone, la jusquiame, la pomme d'amour ; c'est aussi l'opium, le camphre, le nénuphar ... On appliquait ensuite des sangsues sur la région génitale pour apaiser le sexe, prétendument congestionné par le désir sexuel.

Quelques conseils d'hygiène complétaient cette pharmacopée hétéroclite : prendre des bains froids au moins trois fois par jour avec de la limaille et un peu de cannelle et surtout éviter les longs séjours au lit, une couche trop molle, l'inaction, l'oisiveté, les pantalons trop serrés, les amis suspects et les ouvrages licencieux [31].

Le $19 \mathrm{e}$ siècle étant un siècle technique, la vague masturbatoire fut l'occasion de multiples inventions ingénieuses et d'une industrie florissante des appareils préservatifs. Empêcher masturbateurs et masturbatrices de se toucher, pour "sauver leur santé", excita l'esprit bricoleur de petits artisans (Figure 3). Voilà quelques exemples :

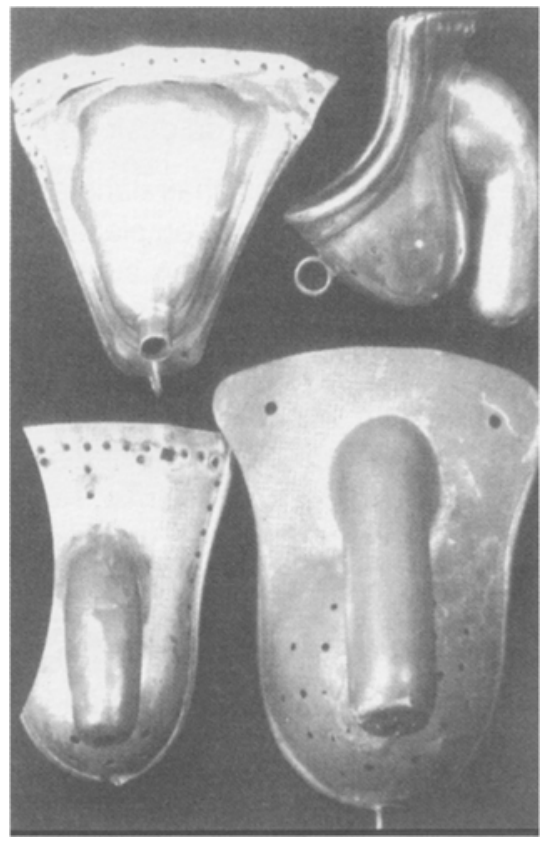

Figure 3 : Appareils anti-masturbatoires.
1) Le fer à cheval (immobilisant le gland sous le sommet de son arceau) et prolongé par deux grelots de métal englobant les testicules - cette ingénieuse trouvaille coercitive s'applique surtout aux adolescents déjà chauds, coutumiers des érections véhémentes. Certains forgerons avaient acquis dans l'art de façonner ces "pièges à canaille" - c'était leur nom, une grande réputation.

2) Les gants cloutés ; les mitaines d'épais mastic (ou d'argile sèche) hérissées d'éclats de verre provenant de bouteilles brisées pour la circonstance.

3) L'horrible venin de serpent, déposé sur le pénis entouré d'un "cylindre" de coton hydrophile (si tu touches, tu t'empoisonnes).

4) Le poignet de force (en acier ou en ciment) qui bloquait l'articulation commandant aux mouvements de la main.

5) La herse en croissant de lune et la cage à barbelés, isolant sous une seule pièce l'appareil sexuel masculin (et féminin) tout entier, anus compris.

6) La camisole de force - comme s'il s'était agit de "réduite" des déments précoces ; mais la coutume de la masturbation ne s'assimilait-elle pas dans l'esprit des châtieurs, à une manifestation de démence juvénile?

7) Le "plat ventre" maintenu par des cordes liant le lit et le supplicié.

8) Les étuis disposés la nuit autour de la verge des garçons "malades". Certains, munis d'une pointe acérée (celle d'un clou !), piquaient le petit vicieux quand survenait une érection obligatoirement coupable, puisqu'on ignorait la physiologie du sommeil paradoxal ; ce pléthysmographe barbare a dû blesser toutes ses victimes [31].

9) Le corset anti-masturbatoire (Figure 4), fabriqué par Jajade-Lafond, bandagiste anti-herniaire. C'était une autre camisole de force, sorte de "ceinture contre l'onanisme", en toile à voile, fermée derrière, soutenue par des bretelles et fermée au dessous à la façon d'un caleçon de bain, afin qu'elle ne pût glisser ni vers le haut ni vers le bas ; à l'extrémité inférieure, dans la région des organes génitaux, se trouvait un petit bouclier de métal de la forme des organes génitaux, donc un long triangle pour les filles et, pour les garçons, une boule creuse destinée à enfermer la verge et les testicules. L'appareil, lacé par-derrière par l'adulte surveillant responsable, incarcérait les organes génitaux externes dans un écusson inviolable. Une petite ouverture comme sur les ceintures de chasteté, laissait passage à l'urine ... Encombrant, vite malodorant, s'imprégnant de sueur et d'urine, contraignant à au moins un coûteux bain hebdomadaire, le carcan n'assura pas la fortune de son inventeur [37].

10) Un dispositif où les mains étaient fixées par de petites serrures à ressort, à une ceinture sur le corps.

11) Un bandage composé d'un tube revêtu de toile, correspondant aux dimensions des parties génitales.

12) Un bandage contre I'"onanisme" qui reposait sur le 


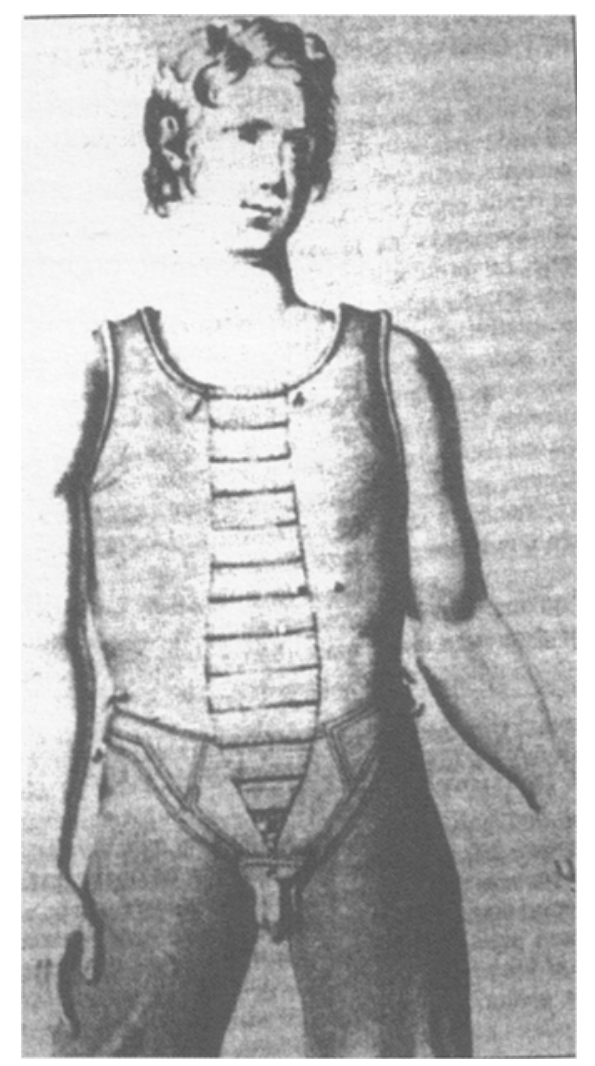

Figure 4 : Le corset contre l'onanisme (1811).

principe de celui de Lafond, mais simplifié : une sorte de bandage herniaire avec deux courroies pour les cuisses, à fermer éventuellement avec trois petits cadenas [31].

13) Des masques de fil de fer, avec des ouvertures minuscules, de manière que ni les doigts ni des objets ne pussent passer au travers pour stimuler les organes génitaux.

14) De petites plaques métalliques, en cuivre ou en fer blanc, étaient appliquées sur les organes sexuels et retenues par des courroies autour des cuisses, pour empêcher le contact des doigts avec les parties ; c'était une imitation des minces plaques de plomb qui devaient être portées dans la région des reins pour combattre les pollutions nocturnes, une idée de Caelius Aurelianus [7].

15) Des clochettes fixées à des bracelets ou à des ceintures de cuir ; le mouvement des mains et donc la manipulation des organes génitaux devaient éveiller le sujet ou son surveillant.

16) Pavet de Courteille recommandait le port de chemises extra-longues qui devaient être attachées sous les pieds. On tenta aussi de lier les mains pour empêcher leur contact avec les organes génitaux.

17) Dans louvrage de J.L.Milton, Spermatorrhea (1887), l'auteur décrit des cages hérissées de pointes qu'on faisait porter la nuit aux malheureux enfants, et même, comble du grotesque, un dispositif électrique qui, à la

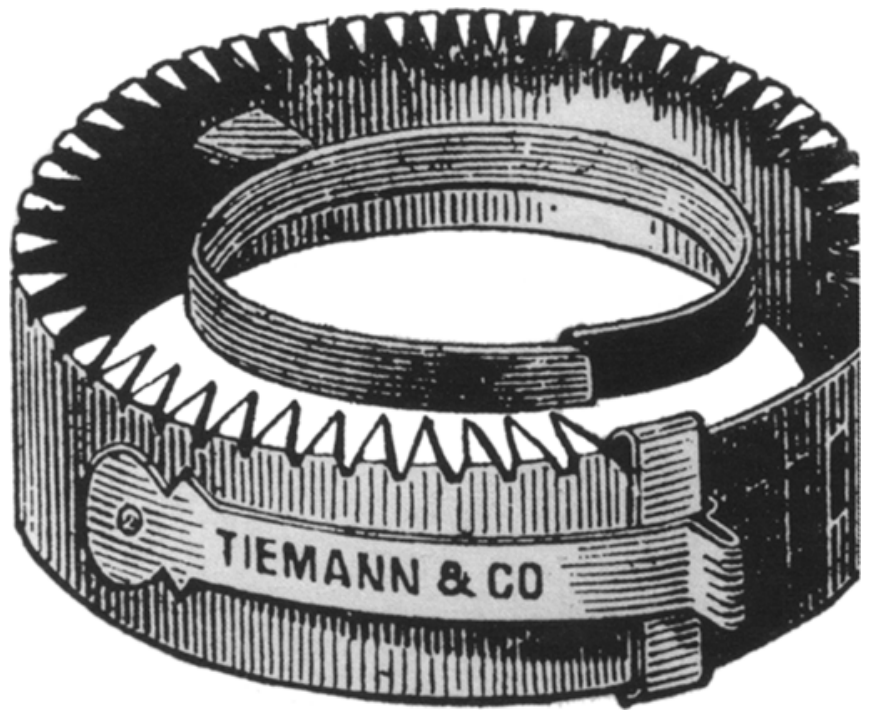

Figure 5 : L'anneau de Tiemann (1876).

moindre érection filiale, déclenchait une sonnerie dans la chambre des parents. Le garçon se réveillait et se lavait à l'eau froide jusqu'à ce que l'érection disparaisse, puis remettait son anneau pour une fin de nuit tranquille.

18) Le Docteur Demeaux déposa le brevet d'un appareil très ingénieux permettant d'aménager des dortoirs spéciaux pour adolescents, dans lesquels la tête et les bras étaient séparés du bas du corps par une solide cloison de bois prévenant toute velléité du désir [37].

19) En 1876, la firme Tiemann affichait dans son catalogue un anneau muni de pointes, que l'on plaçait à la base de la verge (Figure 5). Si une érection survenait au cours du sommeil, les pointes acérées pénétraient dans la peau et la douleur réveillait l'individu [3].

20) Le réveil du Docteur Minière (Figure 6), fabriqué par Collin à Paris, était encore plus perfectionné. La tumescence repoussant une pièce protectrice, faisait entrer la peau en contact avec deux pointes métalliques acérées et reliées à un courant électrique. En même temps qu'il ressentait une douleur due au passage du courant, l'infortuné était réveillé en sursaut par une forte sonnerie électrique. Peu d'érections résistaient à un tel traitement [20].

Cependant, malgré cet arsenal anti-masturbatoire, quelquefois il fallait trouver quelque chose de plus radical en intervenant sur les parties coupables elles-mêmes. 


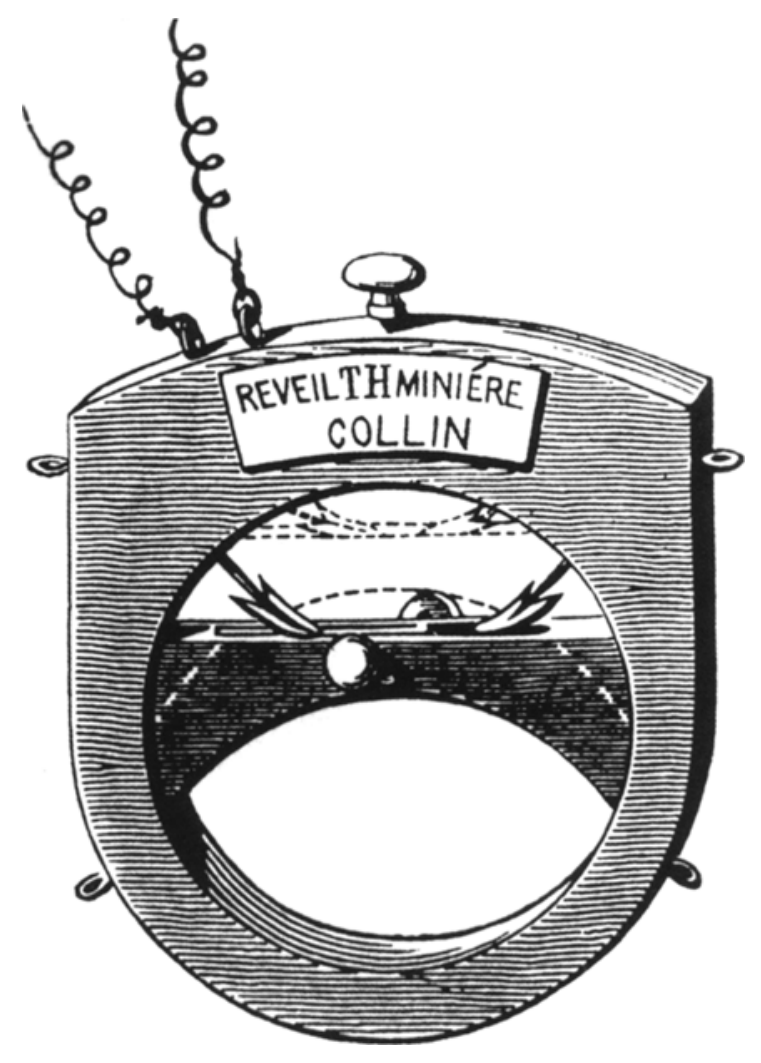

Figure 6 : Le réveil électrique du docteur Minière.

C'est enfin l'électrothérapie et la chirurgie qui donnèrent les meilleurs résultats. Car si I'on ne peut facilement empêcher le geste, on peut beaucoup plus sûrement en supprimer l'objet. La circoncision, la castration, la cautérisation de la région génitale au fer rouge ou à l'électricité ou encore la section des nerfs honteux internes, ceux-là même qui commandent la sensibilité génitale et l'érection, ont été pratiquées en toute méconnaissance de cause et bien que l'on ait remarqué que cette castration n'abolissait par le désir [15].

Pour assagir les masturbateurs on a châtré des "fous". Mais si "la masturbation est un état intermédiaire entre la folie et la raison", il apparut quand même excessif, aux yeux des phallocrates fiers de leurs génitoires, d'envisager la castration et/ou l'émasculation des masturbateurs. II eût fallu châtrer trop de monde. Aussi les tentations interventionnistes à l'encontre de la masturbation masculine demeurèrent-elles velléitaires, réduites à l'état de menaces [16].

Les masturbateurs échappèrent donc au bistouri, sauf quelques cas "gravissimes" de spermatorrhée. On a pu sauver la vie de quelques malheureux menacés d'épuisement complet grâce à la ligature de leurs canaux déférents. La route du sperme était coupée, et les malades ont miraculeusement retrouvé leur embonpoint. On peut d'ailleurs se demander si les chirurgiens n'avaient pas coupé les artères spermatiques par la même occasion, car les opérés ont guéri grâce, entre autres, à une totale disparition de leur désir sexuel [37].

Quant à castration médicale masculine, elle est prescrite pour indication médicale impérative (cancers) et médicolégales répressives (déviance sexuelle). La castration des déviants est une vieille pratique toujours vivace. Honnie par toutes les religions, mais parée de vertus thérapeutiques, la castration est pratiquée jusqu'au 18e siècle par les nombreux opérateurs de hernie qui sillonnent les campagnes, opérant en un tournemain (heureusement unilatéralement) de nombreux enfants et adolescents [34]. Elle est déjà prônée dès l'antiquité dans le traitement de la goutte, de la lèpre, de la chorée, de l'épilepsie, de diverses formes de vésanie, satyriasis, voire même de la manie [1]. Un honorable praticien de Westminster, le Dr Holthouse, pratique en 1859 une première castration infructueuse chez un épileptique ; récidivant dix ans plus tard chez un autre malade de ce type, masturbateur de surcroît, il réussit brillamment. Millant [25] précise "qu'il semble donc qu'il ne faille pas, d'un cas isolé de guérison, conclure d'emblée à la nécessité de la castration pour tous les épileptiques". La manie castratrice est à son comble pendant la période victorienne en Angleterre et aux Etats-Unis (Burns publie en 1896 une statistique de 148 cas de castration pour hypertrophie prostatique) [11].

Lallemand en 1842 publie six volumes sur les pertes séminales involontaires. II les attribue à un état irritatif de la muqueuse de l'urètre postérieur et préconise la cautérisation de la muqueuse prostatique à l'aide de nitrate d'argent, appliqué par une porte caustique [29].

Albert Muller écrit en 1869 un livre sur le même sujet et le dédie à Lallemand ; il déclare dans sa préface que ce dernier pratiqua ses cautérisations quasiment tous les jours durant de nombreuses années et jusqu'à sa mort. On réalise à quel point les pertes séminales, qui dans l'immense majorité des cas ne devaient être que l'expression d'un débordement naturel, provoquaient une profonde inquiétude qui ne tardait pas à déclencher un cortège de manifestations psychosomatiques [14].

\section{CÉLĖBRES MASTURBATEURS}

Galien soulignait les inconvénients de la rétention du sperme et citait en exemple le cas de Diogène qui se masturbait en public!

Samuel Pepys dans son célèbre journal, commencé le 1er janvier 1660 et achevé le 31 mai 1669, soit durant la décennie qui suivit la Restauration et le puritanisme de Cromwell, reconnaît avec une désarmante ingénuité qu'en pleine cérémonie de Noël à la Queen's Chapel de St James, il se masturbe en regardant une jolie fille, et il ajoute: "Que Dieu me pardonne pour l'avoir fait dans la Chapelle", sans y accorder d'autre importance [20].

Curieusement le mot onanisme apparaît dans le vocabulaire français vingt ans après la Révolution. C'est-à-dire sur les traces chaudes de Rousseau qui s'est soulagé plus 
qu'à son tour dans les cabinets et répandu dans les fougères. En fait, Rousseau (mondialement reconnu désormais comme un précurseur de Freud) se livre au vice solitaire. II distrait avec sa main une solitude éveillée sur la rêverie, sur les sens qui la provoquent et l'entretiennent. La pratique de ce vice ne l'a point empêché, notons-le au passage, de faire cinq enfants. Simultanément, la morale publique réprouve haut et fort le plaisir solitaire masculin et, systématiquement, le dramatise en le qualifiant de vice [8].

Denis Diderot en 1782 publia Le Rêve de d'Alembert, dans lequel il fait l'éloge de la masturbation, de "cette chose douce et agréable". En outre, il confessa ce souvenir d'enfance : "Les pages de mon père m'apprirent quelques gentillesses de collège" [21].

Le Marquis de Sade, dont la longue vie carcérale éleva l'acte solitaire au rang de culte et de nécessité, en fait véritablement un art de vivre. L'obsession de la jouissance occupera les jours et les nuits de trente années d'emprisonnement, de trente années de masturbations qui reviennent de façon obsessionnelle dans son œuvre comme le modèle de la jouissance suprême. Dans son premier ouvrage Les cent vingt journées de Sodome, la masturbation est décrite minutieusement [7]. Si Montaigne fut l'inventeur du mot masturbation, de Sade fut le créateur des substantifs qui désignent ses utilisateurs : masturbateur, masturbatrice ...

Des révolutionnaires clament leur liberté et osent avouer la masturbation, ils avouent même s'être fait masturber par des femmes (Baudelaire, Gautier, Maupassant ...) [6].

Nous terminons avec le cas d'Adolf Hitler, qui fut durant les premières périodes de sa vie un masturbateur frénétique. Caporal pendant la guerre 14-18, ils s'était signalé par des extravagances dangereuses dans la boue des tranchées, n'hésitant pas à se masturber debout, la capote relevée, dans les moments les moins propices à de semblables exercices. Savez-vous qu'il avait la manie de conserver son sperme dans une coupe en cristal de Bohème, de le renifler, de monologuer avec lui, de l'admirer sous les cercles grossissants d'une loupe et d'un microscope ? Saviezvous que le futur Chancelier du Reich, lors de sa courte incursion dans le domaine du bâtiment, avait été interpellé sur les échafaudages, faisant pleuvoir sa semence du sommet le plus élevé possible ? Le Führer n'affichait pas de tendances homosexuelles et n'était pas exhibitionniste : il recherchait, pour éjaculer au maximum, les situations les plus inconfortables qui créaient en lui un vertige. Tout cela contredit la partiale légende d'un Hitler "sec", stérile et chichement fourni sur le plan testiculaire. Le sinistre conquérant de l'Europe était un fanatique de Freud dont il avait lu l'œuvre et suivait les travaux. Cette admiration, que personne dans son entourage n'aurait osé lui reprocher, expliquerait sa tolérance compréhensive à l'endroit du maître du Vienne [33].

\section{REFERENCES}

1. ANDROUTSOS G., MARKETOS P. : La Castration à travers les âges. Andrologie, 1993, $1: 61-66$.

2. ANDROUTSOS G., MARKETOS P. : La sexualité dans l'ancienne Egypte. Prog. Urol., 1993, 4 : 715-725.

3. ANDROUTSOS G. : Histoire de la masturbation. Sexologies, $1999,8: 37-44$.

4. BEKKERS : Onania, or the Heinous Sin of Self-pollution and All its Frightful Consequences, in Both Sexes Considered. Crouch, London, 1723.

5. BERNARD P. : Le développement de la personnalité. Paris, Masson, $1973:$ 55-69.

6. BORNEMAN E.R. : La sexologie ; dictionnaire encyclopédique. Verviers, Gérard \& Co, $1973: 232$.

7. BRENOT P.H. : Eloge de la masturbation. Paris, Zulma, 1997 : 11-29.

8. DESLANDES M. : De l'onanisme et des autres abus vénériens. Paris, Le Large, 1835 : 27.

9. EDOUARD R. : Les secrets de la réussite sexuelle féminine. Paris, J. Grancher, $1981:$ 28-23.

10. Encyclopédie de la vie sexuelle. Paris, Hachette, $1973: 145-$ 159;

11. ERLICH M. : La mutilation. Paris, Presses universitaires de France, $1990: 45-50$.

12. FOREL A. : La question sexuelle, exposée aux adultes cultivés. Paris, Steinheil, $1906: 14$.

13. FREUD $S$. : Contributions to the psychology of love ; a neurosis of demoniacal possession in the 17 th century. Hogarth, $1925: 37-38$.

14. GORTER J. : Chirurgia repurgata, Lugduni Batavorum : apud Balduinum. Vander, $1742: 18$.

15. GRODDEK C. : Comportement psychique et traitement psychanalytique des troubles organiques. Leipzig, Hirzel, 1917 : 12.

16. GROSS. D. : A Practical treatise on the urinary organs. Philadephia, Blanchard \& Lea, $1855: 47$.

17. GULIK R. van : La vie sexuelle dans la Chine ancienne. Paris, Gallimard, $1971: 77$

18. FLANDRIN J.L. : L'Église et le contrôle des naissances. Paris, Flammarion, $1970: 20-21$.

19. HIGIER S.T. : Les fonctions sexuelles mâles et leurs troubles. Paris, Gaston Doin, 1932 : 84.

20. KUSS R., GRECOIR W. : Histoire illustrée de l'urologie. Paris, R. Dacosta, 1988 : 464-465.

21. LEWINSOHN R. : Histoire de la vie sexuelle. Paris, Payot, $1957: 23-25$.

22. LOWENFELD W. : De I'onanisme, ou Studien über Atiologie und Pathogenese des spontanen hirnblutungen. Wiesbaden, J. F. Bergmann, $1886: 27-29$.

23. LOWENFELD W. : De la constitution sexuelle et de quelques autres problèmes. Wiesbaden, J. F. Bergmann, $1911: 38$.

24. LUDWIG M. : Lehrbuch der Physiologie des Menschen. Liepzig, Winter, 2 vol., $1858: 61$.

25. MCLAREN A. : Histoire la contraception. Paris, Noêsis, 1996 : 231.

26. MASTERS W., JOHNSON V.C. : Les réactions sexuelles. Paris, Robert Laffont, 1968 : 219-223.

27. MILLANT R. : Les eunuques à travers les âges. Paris, Vigot Frères, 1908 : 161. 
28. MURPHY L. : The history of urology. Springfield, Illinois, Charles Thomas, $1972: 437$.

29. PALAZZOLI M. : Les déficiences génitales chez l'homme. Paris, Masson et Cie, 1951 : 134-140.

30. PELIKAN J. : Luther's Works. Saint-Louis, Concordia, 1965 : $7,20$.

31. SAUVAGE Y. : Les tabous sexuels. Paris, Editions E.P.I., 1983: $20-30$

32. SCHLOSSER L. : L'art ancien de guérir. Paris, France-Empire, $1984 ; 192-195$

33. STEKEL W. : L'homme impuissant. Paris, Gallimard, 1950 : 48.

34. TAYLOR G.R. : Une interprétation sexuelle de l'histoire. Paris, Corréa-Buchet et Chastel, $1954: 250$.

35. TISSOT S.A. : L'onanisme. Paris, ELA La Différence, Paris, 1991: 109-116.

36. VENETTE N. : La génération de l'homme ou tableau de l'amour conjugal. Amsterdam, Ryckhof, 1778.

37. ZWAG G. : Histoire des peines de sexe. Paris, Maloine, 1994 : 228-232.

Manuscrit reçu : décembre 2004 ; accepté décembre 2004

\section{ABSTRACT}

From onanism to masturbation: An historical account

\section{Georges ANDROUTSOS}

This article presents a brief history of masturbation. In particular, subjects such as masturbation in antiquity and the attitude of the church towards this practice are described, along with certain accessories used to facilitate masturbation. Preventive measures used in the past against masturbation are also described, as well as the various cures for the consequences of this vice. The two famous publications Onania and Onanism justifying the polemic against masturbation are also presented.

Key words: masturbation, Onan's crime, Tissot, Bekkers, masturbatory objects, famous onanists 